\title{
The Impact of the COVID-19 Pandemic on Cancer Patients
}

\author{
Osama M. Al-Quteimat, MSc, BCOP* and Amer Mustafa Amer, BSc, MSc $\dagger$
}

\begin{abstract}
In December 2019, a novel coronavirus called severe acute respiratory syndrome coronavirus 2 (SARS-CoV-2) caused an outbreak of coronavirus disease 2019 (COVID-19). Severe complications have been reported to occur in 33\% of patients with COVID-19 and include acute respiratory distress syndrome, acute renal failure, acute respiratory injury, septic shock, and severe pneumonia. Currently, there is no specific treatment or approved vaccine against COVID-19 and many clinical trials are currently investigating potential medications to treat COVID-19. The immunosuppressed status of some cancer patients (whether caused by the disease itself or the treatment) increases their risk of infection compared with the general population. This short review aims to focus on the impact of COVID-19 on a cancer patient and discuss management options and recommendation in addition to highlighting the currently available clinical guidelines and resources.
\end{abstract}

Key Words: COVID-19, cancer, oncology

(Am J Clin Oncol 2020;00:000-000)

\section{CORONAVIRUS DISEASE 2019 (COVID-19)}

Coronaviruses are a large family of viruses that can cause disorders ranging from a mild cold to severe diseases. Some coronaviruses are zoonotic, which means that they spread from animals to humans. In December 2019, a novel coronavirus called severe acute respiratory syndrome coronavirus 2 (SARSCoV-2) caused an outbreak of COVID-19. Typical symptoms of COVID-19 include fever, cough, shortness of breath, and muscle pain. Severe complications have been reported to occur in $33 \%$ of patients with COVID-19 and include acute respiratory distress syndrome, acute renal failure, acute respiratory injury, septic shock, and severe pneumonia. ${ }^{1}$

Currently, there is no specific treatment or approved vaccine against COVID-19, and the clinical management primarily includes implementing recommended infection prevention and control measures and supportive management of complications. Thus far, treatment is provided according to the clinical condition of the patient; supportive treatment such as oxygen therapy, hydration and fever/ pain management, and antibiotics, if bacterial co-infection is present, is recommended. ${ }^{1}$

Many clinical trials are currently investigating potential medications to treat COVID-19 including remdesivir (a prodrug in development), immunoglobulins, arbidol hydrochloride combined with interferon atomization, ASC09F plus oseltamivir, ritonavir plus oseltamivir, lopinavir plus ritonavir and some other drug (https:// clinicaltrials.gov/ct $2 /$ results? cond $=2019$ nCoV\&term $=\&$ cntry $=$ $\&$ state $=\&$ city $=\& d i s t=)$, but no specific treatment or vaccine is approved yet. In addition, chloroquine phosphate (used to prevent

From the *Pharmacy Services Department, Cleveland Clinic Abu Dhabi, Abu Dhabi, UAE; and †Inpatient Pharmacist, The Specialty Hospital, Amman, Jordan.

The authors declare no conflicts of interest.

Reprints: Osama M. Al-Quteimat, MSc, BCOP, Pharmacy Services Department, Cleveland Clinic Abu Dhabi, Abu Dhabi 112412, UAE. E-mail: Alquteo@clevelandclinicabudhabi.ae.

Copyright (c) 2020 Wolters Kluwer Health, Inc. All rights reserved.

ISSN: 0277-3732/20/000-000

DOI: $10.1097 /$ COC.0000000000000712 and treat malaria and some inflammatory conditions) was found to have acceptable safety and demonstrated efficacy in the treatment of COVID-19-associated pneumonia. ${ }^{2}$

\section{COVID-19 AND CANCER}

The immunosuppressed status of some cancer patients (whether caused by the disease itself or the treatment) increases their risk of infection compared with the general population. Immunosuppression may also expose cancer patients to serious complications from an infection, which may result in treatment delay and unnecessary hospitalizations that could negatively affect disease prognosis.

It has been reported by Liang et $\mathrm{al}^{3}$ that patients with cancer have an increased risk of severe infections, with an $\sim 3.5$-fold increase in the risk of needing mechanical ventilation or ICU admission or dying compared with patients without cancer. Cancer patients' increased susceptibility to severe complications of COVID-19 can be attributed to the immunosuppressed status caused by the malignancy and anticancer treatments, such as chemotherapy or surgery. Patients who had received chemotherapy or undergone surgery in the 30 days before presenting with COVID-19 were found to have a higher risk of severe events than patients who had not been treated with chemotherapy or surgery. It was also found that cancer history conferred the highest risk for severe complications and was correlated with poorer outcomes from COVID-19. Notably, lung cancer patients did not have a higher probability of severe complications compared with patients with other cancer types. ${ }^{3}$

Zhang et $\mathrm{al}^{4}$ reported the case of a 57 -year-old Chinese male patient with lung cancer who presented with fever, cough, shortness of breath, myalgia, and diarrhea and later tested positive for COVID-19. The patient's lung cancer was initially treated with gefitinib (an epidermal growth factor receptor [EGFR] inhibitor) starting in February 2016, and the patient was subsequently started on osimertinib monotherapy in September 2017, when the gefinitib resistance-causing mutation EGFR T790M was detected upon disease progression. COVID19 was treated with lopinavir/ritonavir (a combination of protease inhibitors typically used to treat HIV1 infection). Improved pneumonia was reported after 2 weeks of treatment. Three follow-up RT-PCR tests for SARS-CoV-2 were found to be negative, indicating a cure for COVID-19. In the reported case, the patient's clinical condition and performance status permitted continued osimertinib treatment despite the diagnosis of COVID-19.

Wang and Zhang ${ }^{5}$ pointed out that during the COVID-19 pandemic, the primary risk for patients with cancer is limited access to required health care and inability to receive necessary medical services in a timely fashion, especially in high-risk epidemic areas like Wuhan, China, where there is a high demand on medical staff and health care facilities. Health care providers must pay attention to the treatment-related adverse effects in lung cancer patients who are treated with immune checkpoint inhibitors (such as severe myocarditis and pneumonitis): such side effects may negatively affect the patients' survival; thus, it is vital to identify and treat such conditions promptly. 
A recently published retrospective cohort study recruited 28 cancer patients with confirmed COVID-19 from 3 hospitals in Wuhan, China to assess the risk factors associated with ICU admission, mechanical ventilation or death. The study reported that COVID-19-infected cancer patients have a high risk of poor clinical outcomes severe event and mortality.

Cancer treatment within 14 days of COVID-19 diagnosis was reported as a risk factor for developing severe events. Acute respiratory distress syndrome (28.6\%), septic shock (3.6\%), and acute myocardial infarction $(3.6 \%)$ are among the documented severe complications in the study population. Interestingly, $28.6 \%$ of the study patients developed COVID-19 infection while hospitalized which is mainly attributed to the nosocomial transmission. Such findings highlight the importance of implementing strict infection control measures and treating cancer patients in an outpatient setting instead of hospitalization whenever feasible. The authors of this study have recommended that cancer patients currently on cancer treatments should undergo thorough screening for COVID-19 infection and avoid immunosuppressive therapy in case of COVID19 infection. $^{6}$

\section{GUIDELINES AND CLINICAL RESOURCES}

Many clinical resources, including guidelines for COVID19 prevention and management, have recently been published to guide cancer patients, health care professionals, and cancer centers on the proper recommendations and measures to fight this contagious disease and to maximize the use of the available resources in order to sustain health services to cancer patients. One of these resources is a patient information reference issued by the British Columbia Cancer Center that includes many frequently asked questions by cancer patients and offers proper instructions on the required precautions regarding detection of signs and symptoms of COVID-19, social distancing, personal protective equipment use, and hospital visits. ${ }^{7}$ In addition, the Cancer Council of Australia has issued a patient-friendly brochure in many different languages to familiarize cancer patients, including those who are currently being treated, with all of the necessary information regarding COVID-19 and the approved measures of prevention. ${ }^{8}$

Recently, the National Institute for Health and Care Excellence (NICE) issued a clinical guideline for the delivery of systemic anticancer treatments to cancer patients with COVID-19. The NICE guideline provides many recommendations based on the currently available evidence regarding many COVID-19-related and cancerrelated issues, including communicating with patients, managing patients with confirmed or suspected COVID-19, staff who are selfisolating, prioritizing patients for treatment, modifications to usual service, and treatment breaks. Such preliminary guidelines can be helpful tools at this time for health care providers to provide the best available care to cancer patients until more detailed and evidencebased guidelines are available. ${ }^{9}$

In addition, the European Society of Medical Oncology (ESMO) has developed an updated online clinical resource page to highlight all COVID-19-related clinical issues pertaining to cancer. The ESMO clinical resource can provide physicians with proper recommendations based on recent updates about COVID-19 in cancer patients, including information on self-protection, treatment continuity, risk of procedures, and key actions to deliver optimal care to cancer patients in these exceptional circumstances. ${ }^{10}$

The Royal College of Radiologists has also recently established a clinical resource that includes guidelines on the management of many cancers, including breast, upper and lower gastrointestinal, prostate, lung, central nervous system, sarcoma, thyroid, melanoma, gynecologic, and urethral cancers, during the COVID-19 pandemic. These guidelines provide detailed recommendations on treatment with radiotherapy, including types of radiotherapy; administered doses; and when to continue, delay, or stop radiotherapy. ${ }^{11}$

\section{MANAGEMENT AND RECOMMENDATIONS}

Whether to continue cancer therapy or to stop it is still a subject of debate, as the risk of cancer progression after stopping cancer therapy remains controversial. Further clinical trials in cancer patients with COVID-19 are urgently needed before any recommendation can be generalized.

In some solid tumors including lung and pancreatic cancer and hematologic cancers including acute leukemia and high-grade lymphoma, timely diagnosis and treatment are warrantied. While in earlystage diseases such as breast, prostate, cervical, and nonmelanoma skin delaying cancer treatment may be an option during the COVID19 pandemic in high-risk patients.

In general, it is recommended that patients receiving curative cancer therapy should continue their treatment despite the potential risk of COVID-19 infection during anticancer therapy. Delaying treatment of metastatic disease results in deteriorated performance status, admission for symptom palliation, and progressive disease. ${ }^{12}$ Surgeries can be delayed in some cases based on the clinical judgment of the treating physicians. For example, selected early-stage hormone-positive breast cancer patients can continue their hormonal therapy for additional months if needed. Thus, it is recommended that the risks and benefits of treatment delay be assessed for each patient individually in order to identify candidates who would be eligible for treatment delay without major negative health consequences. $^{12}$

Because of the severe clinical deterioration associated with COVID-19 in cancer patients, 3 essential strategies may be used to mitigate the COVID-19 crisis, or any future infection affecting cancer patients. The first strategy is postponing chemotherapy treatment or elective surgical procedures in stable cancer cases at high-risk locations. The second is implementing strict personal precautions in cancer patients or cancer survivors. The third strategy is providing more intensive care and treatment to COVID-19-infected patients with cancer who are old or have other comorbidities. ${ }^{3}$

Online medical counseling and appropriate diagnosis and treatment of critical cases to minimize patients' exposure to COVID-19 may be required during the virus outbreak. ${ }^{5}$ In addition to implementing strict personal precautions to avoid COVID-19 infection, some experts have recommended using outpatient health care facilities to treat non-small cell lung cancer patients with advanced disease whenever feasible during the COVID-19 pandemic. In addition, they recommended ruling out COVID-19 infection before admitting patients to the hospital for cancer therapy. ${ }^{13}$

Kutikov et $\mathrm{al}^{14}$ have proposed specific recommendations which can be used to guide the decision process on delaying or continuing cancer treatment during COVID-19 pandemic. The proposed guide is based mainly on categorizing patients into low, medium or high risk of disease progression with cancer treatment delay. For example; it is considered safe to delay treatment for $>3$ months with low risk of disease progression in patients with chronic hematologic cancers and. Surgery and radiotherapy can also be delayed for the same risk group including patients with nonmelanoma skin cancer, nonlocally advanced breast cancer, low or intermediate-risk prostate cancer, low-grade lymphoma and other low-risk cancer diseases. In contrast, treatment delay is not recommended in patients who are at high risk of disease progression with treatment delay including patients with high grade or aggressive cancers, colon cancer with obstruction, malignancy suspicious ovarian, liver or pancreatic mass and small cell lung cancer ${ }^{14}$ (Table 1$)$. 
TABLE 1. Lists Some Examples on Low and High-risk Conditions Where Cancer Treatment Delay Can Affect Clinical Outcomes ${ }^{14,15}$

\begin{tabular}{ll}
\hline $\begin{array}{l}\text { Low Risk for Complications } \\
\text { With Treatment Delay }\end{array}$ & $\begin{array}{c}\text { High Risk for Complications } \\
\text { With Treatment Delay }\end{array}$ \\
\hline Nonmelanoma skin cancers & $\begin{array}{c}\text { Oncologic emergencies (spinal cord } \\
\text { compression, Hypercalcemia, etc.) }\end{array}$ \\
Chronic hematological cancers & $\begin{array}{l}\text { High-grade sarcoma } \\
\text { Large mass }(>2 \mathrm{~cm} \text { ) lung mass }\end{array}$ \\
$\begin{array}{l}\text { Non grade lymphomas } \\
\text { breast cancers }\end{array}$ & $\begin{array}{l}\text { Acute leukemias and aggressive } \\
\text { lymphomas }\end{array}$ \\
Most thyroid cancers & Head and neck cancers \\
Radiotherapy for low-risk & Chemotherapy for testicular and rectal \\
prostate cancers & cancers \\
Palliative indications & Radiotherapy for gynecologic, rectal, \\
without survival benefits & lung, and neck cancers
\end{tabular}

Treatment prioritization decisions should also consider patient's age, comorbidities and physician's clinical judgment.

\section{INITIATIVES AT HEALTH CARE INSTITUTIONS}

One of the cornerstone strategies for any health care institution to fight COVID-19 is to implement robust infection and environmental control. Identifying and triaging patients suffering from respiratory symptoms is also important to contain SARS-CoV-2 and minimize exposure.

Some health care organizations have adopted strict measures banning travel to certain high-risk areas to protect their facilities and prevent the spread of COVID-19 by. Dana-Farber Cancer Institute and MD Anderson Cancer Center, 2 of the leading health care institutions, have recently banned all business travel to "CDC-defined impacted areas" to limit health care providers' exposure to COVID19. Institutions, along with others, have also encouraged their employees to consider restricting personal travel and to exercise caution during personal time. ${ }^{16}$

Virtual health services, including telephone or online appointments, especially for routine checkups or prescription refills, can also be implemented as appropriate to reduce crowding in health care facilities to reduce exposure and efficiently utilize clinical resources. Moreover, having institutional management guidelines and an emergency plan with equipped isolation areas is required to accommodate any surge in COVID-19 patients.

During this difficult time, it is imperative for any health care organization to address health care providers' well-being and to fulfill their physical and emotional needs by offering the required support to prevent, identify and properly manage any burnout, psychological stress, or safety concerns, especially for those providing direct patient care, such as physicians, respiratory therapists and nurses.

\section{ETHICAL ASPECTS}

During COVID-19 outbreak, many health care systems are facing challenges due to the high demand on pharmaceutical, equipment, and medical supplies that resulted in critical shortages which can force the clinicians to take tough decisions to restrict using advanced therapeutic options including mechanical ventilators to selected patients based on many factors including age, comorbidities or the expected clinical outcomes. Such difficult scenarios may raise ethical considerations regarding the allocation and use of the current limited resources to treat the most critically ill patients with complications or severe conditions. During this crisis, it is vital to apply medical ethics in the clinical practice aiming to treat and cure as many patients as possible with the best use of the available resources.

Rationing treatment, canceling appointments, and delaying surgeries are challenging decisions. In patients with confirmed
COVID-19 and advanced-stage cancer or severe comorbidities requiring mechanical ventilation, poor prognosis is usually expected. In such cases, end-of-life and palliative care plans must be discussed. When faced with limited resources during the COVID-19 pandemic, a cancer treatment multidisciplinary team (which may include medical ethicists and palliative care specialists) must decide which patients will receive complicated or critical care based on the expected clinical outcomes. ${ }^{12}$

The American College of Surgeons (ACS) Committee on Ethics has prepared some guiding principles to help the clinicians and the health care institutions in the decision-making process regarding the allocation of resources during the COVID-19 outbreak. ${ }^{17}$ Such recommendations can be very helpful in resolving the expected ethical dilemma which may affect the emotional health status of the overwhelmed health care providers, due to the high demand for clinical resources and the need to rationalize its use.

Finally, ensuring the continuum of care in cancer patients is vital and is considered a major priority during this time, as many hospitals and health care centers are overwhelmed with increased numbers of COVID-19 cases, high demand on medical supplies (including personal protective equipment), and shortages of health care providers in areas facing an especially high number of cases. Clinical judgment is paramount in determining whether to continue or withhold cancer therapy in patients with suspected or confirmed COVID-19. Health care providers caring for cancer patients are advised to review the latest, rapidly changing literature pertaining to cancer and COVID-19 to provide evidence-based management on a case-by-case basis. There is an urgent need for well-designed trials to identify the clinical consequences of continuing or withholding cancer therapy and the proper prevention, management, and treatment of COVID-19 in the oncology and hematology settings.

\section{REFERENCES}

1. International Pharmaceutical Federation. Coronavirus SARS-CoV2 outbreak: information and guidelines for pharmacists and the pharmacy workforce. Febuary 12, 2020.

2. Gao J, Tian Z, Yang X. Breakthrough: chloroquine phosphate has shown apparent efficacy in treatment of COVID-19 associated pneumonia in clinical studies. BioSci Trends. 2020;14:72-73.

3. Liang W, Guan W, Chen R, et al. Cancer patients in SARS-CoV-2 infection: a nationwide analysis in China. Lancet Oncol. 2020;21: 335-337.

4. Zhang H, Huang Y, Xie C. The treatment and outcome of a lung cancer patient infected with SARS-CoV-2. J Thorac Oncol. 2020. Available at: https://www.jto.org/article/S1556-0864(20)30191-X/ fulltext.

5. Wang H, Zhang L. Risk of COVID-19 for patients with cancer. Lancet Oncol. 2020;21:E181.

6. Zhang L, Zhu F, Xie L, et al. Clinical characteristics of COVID19-infected cancer patients: a retrospective case study in three hospitals within Wuhan, China. Ann Oncol. 2020. Available at: https://www.annalsofoncology.org/article/S0923-7534(20)36383-3/ fulltext.

7. British Columbia Caner Agency. COVID-19 and cancer treatmentsInformation for patients. March 23, 2020. Available at: www.bccancer. bc.ca/about/news-stories/news/2020/covid-19-and-cancer-treatments. Accessed March 27, 2020.

8. Cancer Council Australia. Information and support regarding cancer and COVID-19. Available at: www.cancer.org.au/cancerand-covid-19.html. Accessed March 27, 2020.

9. National Institute for Health and Care Excellence. COVID-19 rapid guideline: delivery of systemic anticancer treatments. March 20, 2020. Available at: https://www.guidelines.co.uk/infection/covid19-rapid-guideline-delivery-of-systemic-anticancer-treatments/455238. article. Accessed March 26, 2020.

10. European Society of Medical Oncology. COVID-19 and cancer. Available at: www.esmo.org/newsroom/covid-19-and-cancer?hit=ehp. Accessed March 26, 2020. 
11. Royal College of Radiologists. Repository of advisory documents for cancer treatment during the coronavirus (COVID-19) pandemic. Available at: www.rcr.ac.uk/college/coronavirus-covid-19-whatrcr-doing/coronavirus-covid-19-resources/coronavirus-covid-19-1. Accessed March 26, 2020.

12. Ueda M, Martins R, Hendrie PC, et al. Managing cancer care during the COVID-19 pandemic: agility and collaboration toward a common goal. J Natl Compr Canc Netw. 2020;18:1-4.

13. Lung Cancer Study Group, Chinese Thoracic Society, Chinese Medical Association, Chinese Respiratory Oncology Collaboration. Expert recommendations on the management of patients with advanced non-small cell lung cancer during epidemic of COVID-19 (Trial version). Chin J Tuberc Respir Dis. 2020; 43:E031.
14. Kutikov A, Weinberg DS, Edelman MJ, et al. A war on two fronts: cancer care in the time of COVID-19. Ann Intern Med. 2020. Doi: 10.7326/M20-1133.

15. Hanna TP, Evans GA, Booth CM. Cancer, COVID-19 and the precautionary principle: prioritizing treatment during a global pandemic. Nat Rev Clin Oncol. 2020. Available at: https://doi.org/ 10.1038/s41571-020-0362-6.

16. Taylor NP. Dana-Farber, MD Anderson ban all business travel over COVID-19. Fiercebiotech. March 6, 2020. Available at: www fiercebiotech.com/biotech/dana-farber-md-anderson-ban-all-businesstravel-over-covid-19. Accessed March 8, 2020.

17. American College of Surgeons. Ethical framework for the allocation of resources in the event of shortages. Available at: www.facs.org/covid-19/ ethics. Accessed April 1, 2020. 